\title{
Three-dimensional dosimetric considerations from different point A definitions in cervical cancer low-dose-rate brachytherapy
}

\author{
Miao Zhang, PhD, Ting Chen, PhD, Leonard H. Kim, DMus, Carl Nelson, MD, Molly Gabel, MD, Venkat Narra, PhD, \\ Bruce Haffty, MD, Ning J. Yue, PhD \\ Department of Radiation Oncology, The Cancer Institute of New Jersey, New Brunswick, New Jersey, USA
}

\begin{abstract}
Purpose: To investigate the dosimetric difference due to the different point A definitions in cervical cancer lowdose-rate (LDR) intracavitary brachytherapy.

Material and methods: Twenty CT-based LDR brachytherapy plans of 11 cervical patients were retrospectively reviewed. Two plans with point As following the modified Manchester system which defines point A being $2 \mathrm{~cm}$ superior to the cervical os along the tandem and $2 \mathrm{~cm}$ lateral $\left(\mathrm{A}_{\mathrm{os}}\right)$, and the American Brachytherapy Society (ABS) guideline definition in which the point $A$ is $2 \mathrm{~cm}$ superior to the vaginal fornices instead of os $\left(A_{\text {ovoid }}\right)$ were generated. Using the same source strength, two plans prescribed the same dose to $A_{o s}$ and $A_{o v o i d}$. Dosimetric differences between plans including point A dose rate, treatment volume encompassed by the prescription isodose line (TV), and dose rate of $2 \mathrm{cc}$ of the rectum and bladder to the prescription dose were measured.

Results: On average $\mathrm{A}_{\text {ovoid }}$ was $8.9 \mathrm{~mm}$ superior to $\mathrm{A}_{\mathrm{os}}$ along the tandem direction with a standard deviation of $5.4 \mathrm{~mm}$. With the same source strength and arrangement, $A_{\text {os }}$ dose rate was $19 \%$ higher than $A_{\text {ovoid }}$ dose rate. The average $\mathrm{TV}\left(\mathrm{A}_{\text {ovoid }}\right)$ was $118.0 \mathrm{cc}$, which was $30 \%$ more than the average $\mathrm{TV}\left(\mathrm{A}_{\mathrm{os}}\right)$ of $93.0 \mathrm{cc} . \mathrm{D}_{2 \mathrm{cc}} / \mathrm{D}\left(\mathrm{A}_{\text {prescribe }}\right)$ increased from $51 \%$ to $60 \%$ for rectum, and increased from $89 \%$ and $106 \%$ for bladder, if the prescription point changed from $\mathrm{A}_{\text {os }}$ to $\mathrm{A}_{\text {ovoid. }}$.

Conclusions: Different point A definitions lead to significant dose differences. Careful consideration should be given when changing practice from one point A definition to another, to ensure dosimetric and clinical equivalency from the previous clinical experiences.
\end{abstract}

Key words: cervical cancer, LDR brachytherapy, point A.

\section{Purpose}

Intracavitary brachytherapy is an integral component of definitive treatment for locally advanced cervical cancer. Traditionally, two dimensional (2D) film-based planning with point dose-based prescription was used [1], in which dose is prescribed to the point $\mathrm{A}$, and the isodose line traveling through point A forms the classic pear shape encompassing the intended boost treatment volume. With increasing availability of 3-dimensional (3D) imaging compatible applicators, 3D volumetric planning has been recommended by the American Brachytherapy Society (ABS) 2012 guidelines [2-4]. By contouring the high risk clinical target volume (HR-CTV), the bladder and the rectum dose volume information, e.g., $\mathrm{D}_{90 \%}$ of the HR-CTV, and the $\mathrm{D}_{2 \mathrm{cc}}$ of the rectum and the bladder, would be used to design the plan. In clinical practice, due to the limited access of good soft tissue contrast image modality, e.g., MRI, a hybrid planning approach combining point A-based prescription and dose volume-based plan evaluation is often adopted, in which CT images are used to localize sources and evaluate critical structure dose volume information, but point A-based prescription rather than target-based dose prescription is used. The abundant clinical experience accumulated by the radiation oncologists throughout decades of practice, makes point A-based prescription still an important component in cervical cancer brachytherapy with active researches underway [5-7].

The definition of point $\mathrm{A}$ has had several variations in terms of its location along the tandem direction. In the original Manchester system [1], point A was defined as " $2 \mathrm{~cm}$ lateral to the central canal of the uterus, and $2 \mathrm{~cm}$ up from the mucous membrane of the lateral fornix in the 
axis of the uterus" ( $\left.\mathrm{A}_{\text {ovoid }}\right)$. In 1953, the definition was modified to be " $2 \mathrm{~cm}$ up from the lower end of the last intrauterine tube, and then $2 \mathrm{~cm}$ laterally in the plane of uterus" $\left(\mathrm{A}_{\mathrm{os}}\right)$ based on the observation that a high proportion of the patients had "the cervix eroded away and the lateral vaginal fornices covered by fungating tumor or, in the indurated type of cancer, so narrowed that they scarcely exist" [8]. Since the last intrauterine tube is usually placed against the cervical os, following this definition $\mathrm{A}_{\mathrm{os}}$ would be easier to define in 2D film-based planning due to the superior radiographic visibility of the flange of the tandem, which is supposedly adjacent to the cervical os. These two definitions of point A are both used clinically and described in textbooks. For example, the definition of point $A$ from the vaginal fornices $\left(\mathrm{A}_{\text {ovoid }}\right)$ has been adopted by the ABS guidelines [2-4], whereas the definition of point $A$ from the cervical os $\left(A_{o s}\right)$ is described and referenced to in a standard medical physics textbook [9].

Switching between two point A definitions in clinical practice may be problematic. Several studies [5,10-15] showed that a slight variation in point A location can result in significant dose variation. In a very recent study, Anderson et al. [5] evaluated the impact of selection of different point As on the dose to HR-CTV contoured from MR images in high-dose-rate (HDR) cervical cancer brachytherapy patients. They found on average there were small difference between two different point A definitions. However, in certain cases the point A dose difference could be as high as $12 \%$. In this study, we retrospectively evaluated the dosimetric impacts due to different point A definitions based on a series of 3D CT planning images for low-dose-rate (LDR) cervical cancer brachytherapy from our institution. In addition to the geometric shift of point A location from one definition to another, the changes of the treatment volume (i.e., the volume encompassed by the prescription isodose line) and the changes of various volumetric, and dosimetric parameters of critical organs were also analyzed. Results derived from this study may provide useful information on relationships between point A definitions and 3D volumetric, and dosimetric parameters and may help in the transfer of clinical experiences of point A-based prescription to 3D target volume-based prescription.

\section{Material and methods}

Twenty CT-based LDR brachytherapy plans of 11 cervical patients treated from December 2009 through August 2011 were retrospectively reviewed in the Eclipse ${ }^{\circledR}$ treatment planning system (Varian Medical Systems, Palo Alto, CA). The hybrid approach was used in all treatment plans. Critical organs including the rectum and the bladder were contoured and confirmed by two radiation oncologists independently. In the original plan used for treatment, point As were defined following the modified Manchester definition $\left(\mathrm{A}_{\mathrm{os}}\right): 2 \mathrm{~cm}$ superior to the cervical os along the tandem and $2 \mathrm{~cm}$ lateral. The location has been double checked by two physicists independently. In practice, the top of the flange on the tandem was used as a surrogate for the cervical os since the anatomical os was difficult to identify and the flange was right next to the os. In all cases, Henschke applicators [16] were used with different cap sizes, and 3-4 ${ }^{137}$ Cs sources were used in the tandem and $1{ }^{137} \mathrm{Cs}$ source in each ovoid. The source arrangement was optimized to deliver prescription dose to point $A$ while minimizing the values of $\dot{D}_{2 c c}$ (the dose rate value corresponding to $2 \mathrm{cc}$ on the cumulative dose volume histogram) of the rectum and the bladder. The prescription dose to point A was 22.5 Gy for each fraction following our institution guideline for cervical cancer LDR.

$\mathrm{A}_{\text {ovoid }}$ was inserted into the same CT image following the 2012 ABS recommendation [2] for each of the clinical plans. A new plan was generated based on the same source arrangement, but with the prescription point changed from $A_{\text {os }}$ to $A_{\text {ovoid. }}$. It should be noted that since two point As are most likely at different locations with different dose rates, the change of the prescription point would change the treatment time, and the absolute dose to the target volumes and normal structures. The amount of geometric shift between two definitions of point As was measured. Dosimetric parameters including dose rates to both point As, the treatment volumes (TV) and dose rates to $2 \mathrm{cc}$ of the rectum and the bladder respect to the prescription dose $\left(\dot{D}_{2 c c} / \dot{D}(A)\right)$ were recorded. TV was defined as the volume encompassed by the isodose rate line traveling through the prescription point, e.g., in the original plan it was the volume encompassed by isodose rate line traveling through $\mathrm{A}_{\mathrm{os}}$, and in the new plan it was the volume encompassed by isodose rate line traveling through $\mathrm{A}_{\text {ovoid }}$.

\section{Results}

A histogram of the shifts between $A_{\text {ovoid }}$ and $A_{o s}$ is shown in Figure 1. A positive value on the $X$-axis indicates that $A_{\text {ovoid }}$ was superior to $A_{\text {os }}$ along the tandem direction. The average shift between $\mathrm{A}_{\text {ovoid }}$ and $\mathrm{A}_{\mathrm{os}}$ was $8.9 \mathrm{~mm}$ with a standard deviation of $5.4 \mathrm{~mm}$. Only 2 out of 20 cases had $\mathrm{A}_{\text {ovoid }}$ inferior to $\mathrm{A}_{\mathrm{os}}$.

Based on the original planned source arrangement which was kept to be the same between the original clinical plan and the new plan, the average dose rate at $\mathrm{A}_{\text {ovoid }}$ was $43.7 \mathrm{cGy} / \mathrm{hr}$, and the average dose rate at $\mathrm{A}_{\mathrm{os}}$ was $51.3 \mathrm{cGy} / \mathrm{hr}$. The standard deviation of the dose rate at $\mathrm{A}_{\text {ovoid }}$ was $6.38 \mathrm{cGy} / \mathrm{hr}$ (14.6\% of the mean) and $6.77 \mathrm{cGy} /$ $\mathrm{hr}\left(13.2 \%\right.$ of the mean) for $\mathrm{A}_{\text {os }}$. If we calculate $\left(\mathrm{D}\left(\mathrm{A}_{\text {os }} /\right.\right.$ $\dot{\mathrm{D}}\left(\mathrm{A}_{\text {ovoid }}\right)$ for an individual case, the dose rate ratio was $119 \%$ on average. The average treatment volume (TV) defined by $\mathrm{A}_{\text {ovoid }}\left(\mathrm{TV}\left(\mathrm{A}_{\text {ovoid }}\right)\right)$ was $118.0 \mathrm{cc}$, which was $30 \%$ more than the average TV $\left(A_{o s}\right)$ of $93.0 \mathrm{cc}$. Table 1 listed the treatment volume (TV) between the original clinical plan and the new plan among all the cases.

Figure 2 shows the relationships between the geometric shift of point $A\left(A_{\text {ovoid }}-A_{o s}\right)$, the ratio of $\dot{D}\left(A_{\text {ovoid }} /\right.$ $\dot{\mathrm{D}}\left(\mathrm{A}_{\mathrm{os}}\right)$ and $\mathrm{TV}\left(\mathrm{A}_{\text {ovoid }}\right) / \mathrm{TV}\left(\mathrm{A}_{\mathrm{os}}\right)$. With point $\mathrm{A}$ shifting away from the ovoids, $\dot{D}(\mathrm{~A})$ decreased and TV(A) increased. From the graph, the geometric shift of point A was poorly correlated with the dose rate change to point $\mathrm{A}$ and TV change. Therefore, there was no reliable mathematical formula to calculate the dosimetric consequences, e.g., point A dose rate change, and the TV change, from the geometric changes. 
Table 1. Dosimetric parameters between the original plan and the new plan

\begin{tabular}{|c|c|c|c|c|c|c|}
\hline \multirow[t]{2}{*}{ Case \# } & \multicolumn{3}{|c|}{ Original plan $\left(\mathrm{A}_{\mathrm{os}}\right)$} & \multicolumn{3}{|c|}{ New plan $\left(\mathrm{A}_{\text {ovoid }}\right)$} \\
\hline & $\mathrm{TV}[\mathrm{cc}]$ & $\mathrm{D}_{\text {rectum }}[\%]$ & $\mathrm{D}_{\text {bladder }}[\%]$ & $\mathrm{TV}[\mathrm{cc}]$ & $\mathrm{D}_{\text {rectum }}[\%]$ & $\mathrm{D}_{\text {bladder }}[\%]$ \\
\hline 1 & 91.3 & 26 & 86 & 101.4 & 28 & 92 \\
\hline 2 & 122.9 & 36 & 120 & 120.0 & 35 & 118 \\
\hline 3 & 85.6 & 46 & 51 & 127.5 & 60 & 67 \\
\hline 4 & 78.5 & 60 & 82 & 85.1 & 64 & 86 \\
\hline 5 & 82.6 & 44 & 78 & 111.7 & 54 & 96 \\
\hline 6 & 85.3 & 44 & 83 & 83.3 & 44 & 81 \\
\hline 7 & 108.8 & 59 & 85 & 131.4 & 67 & 97 \\
\hline 8 & 106.9 & 50 & 92 & 115.6 & 52 & 97 \\
\hline 9 & 88.3 & 55 & 96 & 105.2 & 62 & 108 \\
\hline 10 & 68.2 & 84 & 112 & 87.8 & 100 & 133 \\
\hline 11 & 102.7 & 71 & 81 & 103.8 & 72 & 82 \\
\hline 12 & 114.5 & 57 & 96 & 135.4 & 64 & 107 \\
\hline 13 & 70.7 & 38 & 88 & 126.6 & 57 & 130 \\
\hline 14 & 97.2 & 40 & 111 & 140.0 & 51 & 142 \\
\hline 15 & 71.1 & 37 & 76 & 104.5 & 48 & 98 \\
\hline 16 & 97.8 & 60 & 72 & 117.5 & 68 & 82 \\
\hline 17 & 93.1 & 62 & 120 & 138.3 & 81 & 156 \\
\hline 18 & 121.2 & 61 & 73 & 114.3 & 58 & 70 \\
\hline 19 & 65.2 & 38 & 88 & 154.6 & 69 & 159 \\
\hline 20 & 107.6 & 45 & 99 & 155.1 & 57 & 127 \\
\hline Average & 93.0 & 51 & 89 & 118.0 & 60 & 106 \\
\hline
\end{tabular}

${ }^{*} T V$ is the treatment volume. Rectum and bladder dose is specified as $D_{2 c c} D_{A}$.

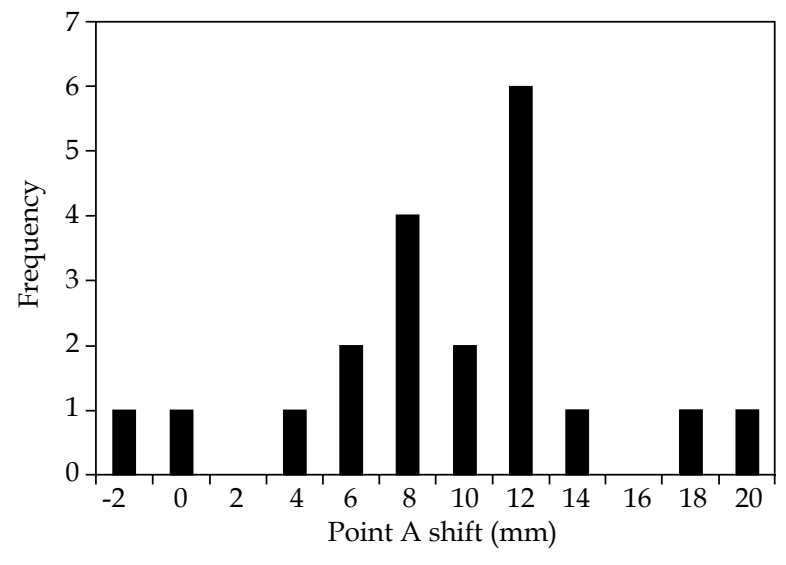

Fig. 1. A histogram of the shifts between $A_{\text {ovoid }}$ and $A_{o s}$ along the direction parallel to the tandem. The positive number indicates $\mathrm{A}_{\text {ovoid }}$ was superior to $\mathrm{A}_{\mathrm{os}}$

The doses to $2 \mathrm{cc}$ of the rectum and the bladder were measured relative to the prescription dose and listed in Table 1. With the same source arrangement between the original clinical plan and the new plan, the isodose-rate lines were fixed. Therefore, $\dot{\mathrm{D}}_{2 \mathrm{cc}}$ for bladder and rectum would stay the same regardless of the prescription me- thod. However, by prescribing to point $\mathrm{A}$, the bladder and rectum $D_{2 c c}$ would be different between two plans and determined by the $\dot{\mathrm{D}}_{2 \mathrm{cc}}$ to $\dot{\mathrm{D}}(\mathrm{A})$ ratio. In the original plan which had $\mathrm{A}_{\mathrm{os}}$ as the prescription point, the average rectum and the bladder $\dot{\mathrm{D}}_{2 \mathrm{cc}}$ were $51 \%$ and $89 \%$ of $\dot{\mathrm{D}}\left(\mathrm{A}_{\mathrm{os}}\right)$, respectively, compared to $60 \%$ and $106 \%$ of $\dot{D}\left(A_{\text {ovoid }}\right)$, when $\mathrm{A}_{\text {ovoid }}$ was used as the prescription point.

\section{Discussion}

In this study we were able to utilize 3D CT imageguided treatment planning to extract information about not only the shift of point $\mathrm{A}$ and dose rate change, but also the changes of prescribed treatment volume and critical organ volumetric dose in LDR cervical cancer brachytherapy. This study revealed that there was a clear difference in dose delivered when using the two different point A definitions. For the investigated patient population, this difference led to an almost $9 \mathrm{~mm}$ shift in point $\mathrm{A}$ location along the tandem on average, which is similar to Anderson et al.'s finding [5]. This observation is different from the finding by Tod and Meredith [8], in which they suggested the change from the original point A definition in the Manchester system $\left(\mathrm{A}_{\text {ovoid }}\right)$ to the modified Manchester system $\left(\mathrm{A}_{\mathrm{os}}\right)$ due to minimum difference between those two points, but better visualization of $A_{o s}$ on radio- 
graph. They wrote: "in the average case the lower end of the cervical canal is level with the lateral fornices, as indicated by ovoid position". The difference in the current study could be due to the difference in patients' bulk of disease at the time of treatment. In their paper, the authors mentioned that a high proportion of patient under treatment had their cervix "eroded away". Patients with such an advanced stage of disease may not be as common in today due to comprehensive screening and early diagnoses. Although the original rationale for changing the original point A definition in the Manchester system $\left(\mathrm{A}_{\text {ovoid }}\right)$ to the modified Manchester system $\left(\mathrm{A}_{\mathrm{os}}\right)$ may not apply today, we are not recommending one definition over another, which is a question that should only be answered by clinical trials.

This study is intended to provide volumetric and dosimetric information on what a clinician may encounter if he/she utilizes the different definition of point $A$ in clinical practice. Currently, with the recently published ABS guidelines [2-4], it is likely that more and more practices which have been prescribing to $A_{o s}$ will tend to start to prescribe to $\mathrm{A}_{\text {ovoid. }}$. If the source arrangement and source strength remains the same, the dose rate at $\mathrm{A}_{\text {ovoid }}$ is $14 \%$ lower than that of $\mathrm{A}_{\mathrm{os}}$. Therefore, without correspondingly adjusting the prescription dose, the total reference air kerma would be $14 \%$ higher and the treatment volume would be $30 \%$ larger. This finding is larger than Anderson et al.'s finding, in which the change of total reference air kerma and treatment volume were merely $2 \%$ and 3\%, respectively. The difference may due to the difference treatment modalities, e.g., LDR vs. HDR.

As shown in the current study, with a same source arrangement and strength, the dose rate at $\mathrm{A}_{\text {ovoid }}$ is likely to be lower than that at $A_{o s}$. In clinical practice, it is often the case where the source strength and arrangement is chosen, so that the dose rate at the prescription point is close to a certain value. Thus, if a decision is made to shift the prescription point from $\mathrm{A}_{\mathrm{os}}$ to $\mathrm{A}_{\text {ovoid }}$ and the dose rate at $\mathrm{A}_{\text {ovoid }}$ is made to be similar to the value previously used for the cases with $A_{o s}$ as the prescription point, the resultant dose rate at $A_{o s}$ is most likely going to be higher, indicating a higher dose rate delivery compared to the prior clinical treatments. To demonstrate this effect, we carried out a simple experiment: a new plan prescribing to $\mathrm{A}_{\text {ovoid }}$ was generated assuming its prescription point $A_{\text {ovoid }}$ received the same dose rate as the prescription point $A_{\text {os }}$ had in the original plan. Figure 3 compares the dose rate to $\mathrm{A}_{\mathrm{os}}$ in the original plan and the dose rate to $A_{o s}$ in the new plan. In the original plan, the dose rate to $A_{o s}$ was $51.3 \mathrm{cGy} / \mathrm{hr}$ on average, whereas in the new plan the dose rate to $\mathrm{A}_{\mathrm{os}}$ had an average of 61.7 cGy/hr with certain cases well beyond 80 cGy/hr. As pointed out in the ABS guideline for LDR cervical cancer brachytherapy [4], when point A dose rate increased from a typical 40-60 cGy/hr LDR range to $80-120$ cGy/hr of medium-dose-rate range, late complications, including small bowel obstruction, vesico-vaginal fistula, and ureterohydronephrosis could increase from $30 \%$ to $45 \%$, even the prescription dose remains the same [17]. Thus, when the prescription point is changed, both total prescription dose and dose rate to the previously would be defined

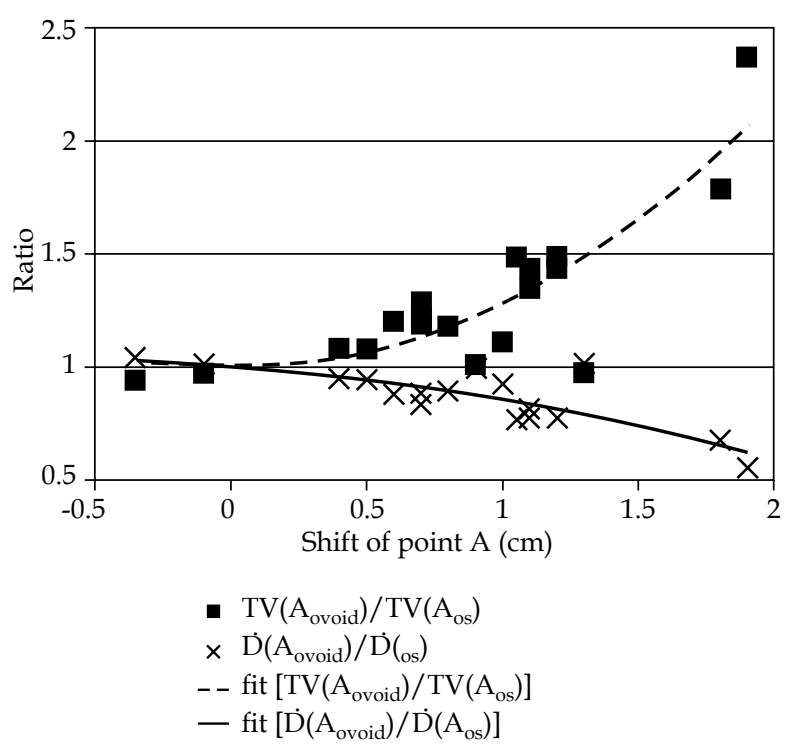

Fig. 2. Shift between $A_{\text {os }}$ and $A_{\text {ovoid }}$ vs. $\dot{D}\left(A_{\text {ovoid }}\right) / \dot{D}\left(A_{\text {os }}\right)$ and $\operatorname{TV}\left(\mathrm{A}_{\text {ovoid }}\right) / \operatorname{TV}\left(\mathrm{A}_{\mathrm{os}}\right)$. The $2^{\text {nd }}$ order polynomial fitting lines are also plotted

treatment volume have to be taken into consideration to replicate accumulated clinical experiences.

Point A based cervical cancer brachytherapy prescription and reporting has been introduced along with the film based planning in order to provide consistency in dose delivery and reporting, allowing for easier comparison of clinical outcomes. With the increasing availability of the 3D CT images, utilization of film based planning may diminish. However, point A based prescription and reporting is still encouraged as the most current clinical experience is based on this practice and the prescription can be easily integrated in the 3D image based planning. This study further demonstrated that the point A re-defi-

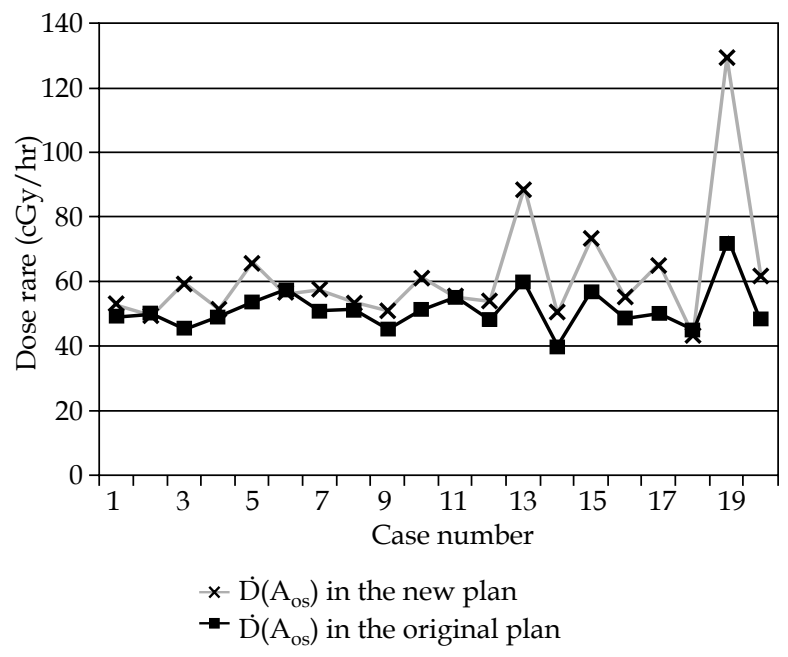

Fig. 3. Dose rate to $A_{o s}$ in the original plan (prescribed to $A_{o s}$ ) and the new plan (prescribed to $A_{\text {ovoid }}$ ), which delivers the same dose rate to the prescription point of all 20 cases 
nition can lead to significant dosimetric differences. Careful consideration should be given when changing practice from one point A definition to another to ensure dosimetric, and clinical equivalency from the previous clinical experiences.

\section{Disclosure}

Authors report no conflict of interest.

\section{References}

1. Tod MC, Meredith W. A dosage system for use in the treatment of cancer of the uterine cervix. Br J Radiol 1938; 11: 809-824.

2. Viswanathan AN, Thomadsen B. American Brachytherapy Society consensus guidelines for locally advanced carcinoma of the cervix. Part I: General principles. Brachytherapy 2012; 11: 33-46.

3. Viswanathan AN, Beriwal S, De Los Santos JF et al. American Brachytherapy Society consensus guidelines for locally advanced carcinoma of the cervix. Part II: High-dose-rate brachytherapy. Brachytherapy 2012; 11: 47-52.

4. Lee LJ, Das IJ, Higgins SA et al. American Brachytherapy Society consensus guidelines for locally advanced carcinoma of the cervix. Part III: Low-dose-rate and pulsed-dose-rate brachytherapy. Brachytherapy 2012; 11: 53-57.

5. Anderson J, Huang Y, Kim Y. Dosimetric impact of point A definition on high-dose-rate brachytherapy for cervical cancer: evaluations on conventional point $\mathrm{A}$ and MRI-guided, conformal plans. J Contemp Brachytherapy 2012; 4: 241-246.

6. Tyagi K, Mukundan H, Mukherjee D et al. Non isocentric film-based intracavitary brachytherapy planning in cervical cancer: a retrospective dosimetric analysis with CT planning. J Contemp Brachytherapy 2012; 4: 129-134.

7. Opfermann KJ, Wahlquist A, Watkins J et al. Impact of point A asymmetry on local control and survival for low dose-rate (LDR) brachytherapy in cervical cancer. J Contemp Brachytherapy 2012; 1: 3-7.

8. Tod M, Meredith W. Treatment of cancer of the cervix uteri-A revised "Manchester method". Br J Radiol 1953; 26: 252-257.

9. Khan F. The Physics of Radiation Therapy. 4 ed. Lippincott Williams \& Wilkins, Baltimore 2009; pp. 315-353.

10. Potish R, Gerbi B. Role of point A in the era of computerized dosimetry. Radiology 1986; 158: 827-831.

11. Kim Y, Huang Y, Bayouth J et al. Dosimetric Consequences of the Prescription Point $\mathrm{H}$ of ABS Recommendation in the Era of MRI Guided Brachytherapy for Cervical Cancer: Based on GYN GEC-ESTRO Recommendations of MRI Guided Brachytherapy. Int J Radiat Oncol Biol Phys 2008; 72: S586-S587.

12. Howell R, Prete J, Wiatrowski W et al. Calculation/comparison of dose to point " $\mathrm{H}$ " according to the ABS recommendations for HDR brachytherapy for carcinoma of the cervix to the dose to point " $\mathrm{A}$ " based on the revised manchester system. Int J Radiat Oncol Biol Phys 2001; 51: 330-331.

13. Eng T, Cummins S, Baake D. Point A or Point H in Prescribing High-Dose-Rate (HDR) Intracavitary Brachytherapy for Cervical Carcinoma? Int J Radiat Oncol Biol Phys 2007; 69: S396-S397.

14. Demandante C, Eng T, Yam M et al. Comparison of Highdose Rate (HDR) Brachytherapy Dose-prescription to Point A versus to Point $\mathrm{H}$ in the Treatment of Cervical Carcinoma. Int J Radiat Oncol Biol Phys 2008; 72: S364-S365.

15. Chennupati S, Merz B, Kalpathy-Cramer J et al. Variations in Point Dose Specification in 3-Dimensional Planning for Intracavitary Cervical Brachytherapy. Int J Radiat Oncol Biol Phys 2011; 81: S469-S470.
16. Henschke UK, Hilaris BS, Mahan G. Remote afterloading with intracavitary applicators. Radiology 1964; 83: 344-345.

17. Haie-Meder C, Kramar A, Lambin P et al. Analysis of complications in a prospective randomized trial comparing two brachytherapy low dose rates in cervical carcinoma. Int $\mathrm{J} R \mathrm{R}$ diat Oncol Biol Phys 1994; 29: 953-960. 